XXVII International Conference on Photonic, Electronic and Atomic Collisions (ICPEAC 2011) IOP Publishing Journal of Physics: Conference Series 388 (2012) 032055 doi:10.1088/1742-6596/388/3/032055

\title{
Above-threshold ionization in atomic hydrogen using intense, few-cycle laser pulses
}

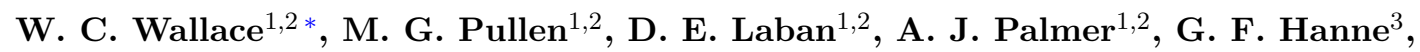
A. N. Grum-Grzhimailo ${ }^{4,5}$, B. Abeln ${ }^{4}$, K. Bartschat ${ }^{4}$, D. Weflen $^{4}$, I. Ivanov ${ }^{6}$, A. Kheifets ${ }^{6}$, H. M. Quiney ${ }^{7}$, I. V. Litvinyuk ${ }^{2}$, R. T. Sang ${ }^{1,2}$, and D. Kielpinski ${ }^{1,2}$

${ }^{1}$ ARC Centre of Excellence for Coherent X-Ray Science, Griffith University, Nathan, QLD, 4111, Australia

${ }^{2}$ Australian Attosecond Science Facility and Centre for Quantum Dynamics, Griffith University, Nathan, QLD, 4111, Australia

${ }^{3}$ Atomic and Electronics Physics Group, Westfälische Wilhelms-Universität, Münster, Germany

${ }^{4}$ Department of Physics and Astronomy, Drake University, Des Moines, Iowa, USA

${ }^{5}$ Institute of Nuclear Physics, Moscow State University, Moscow, Russia

${ }^{6}$ Research School of Physical Sciences, The Australian National University, Canberra, ACT, Australia

${ }^{7}$ ARC Centre of Excellence for Coherent X-Ray Science, University of Melbourne, Melbourne, VIC, Australia

\begin{abstract}
Synopsis The interaction of intense few-cycle light pulses and matter has given rise to a number of high-field physical processes that are of great interest. The high non-linearity of such interactions necessitates complex numerical simulations in order to retrieve useful physical measurements from the experimental data. Here we demonstrate quantitative agreement at the $10 \%$ level between experimentally obtained integrated photoelectron spectra and numerical simulations for above-threshold ionization in the intense few-cycle regime. The use of atomic hydrogen provides a key experimental innovation as it is the only electronic system for which ab initio simulations in this regime are available.
\end{abstract}

Above-threshold ionization (ATI) is the process whereby an atom can absorb more photons than the minimum number required for ionization to occur [1]. Since its discovery by Agostini et al in 1979 [2], many studies on the process of ATI have been done both in theory and experiment. In the few-cycle regime, agreement between theory and experiment has been qualitative at best. We demonstrate the first experiment in ATI of atomic hydrogen using few-cycle pulses that shows an unprecedented level of quantitative agreement between experiment and theory.

The experimental apparatus consists of a linearly polarized few-cycle strong-field laser, an atomic $\mathrm{H}$ beam and a detection system. Laser pulses of energy $150 \mu \mathrm{J}, \sim 6.0$ fs duration and 1 $\mathrm{kHz}$ repetition rate intersect and ionize the orthogonally oriented $\mathrm{H}$ beam. The $\mathrm{H}$ beam is created via collisional dissociation of $\mathrm{H}_{2}$ in to $\mathrm{H}$ via a radio frequency discharge. Photoelectrons ejected from the interaction region traverse a series of electrostatic lenses which act to repel low energy electrons before being accelerated to $\sim 250$ $\mathrm{eV}$ for detection by a channeltron. Processing of the channeltron signal produces the integrated photoelectron yield as a function of the retarding voltage applied to the electrostatic lenses.

Recently submitted work of the comparison between the experimental data and theoretical data simulated from direct integration of the non-relativistic time-dependent Schrodinger equation (TDSE) is shown in Figure 1. This re-

\footnotetext{
${ }^{*}$ E-mail: wc.wallace@gmail.com
}

markable quantitative agreement is achieved over a wide range of electron energies and laser intensities and shows the critical dependence on the implementation of the theoretical models. We have also investigated the effect of laser chirp on the ejected photoelectron yield.

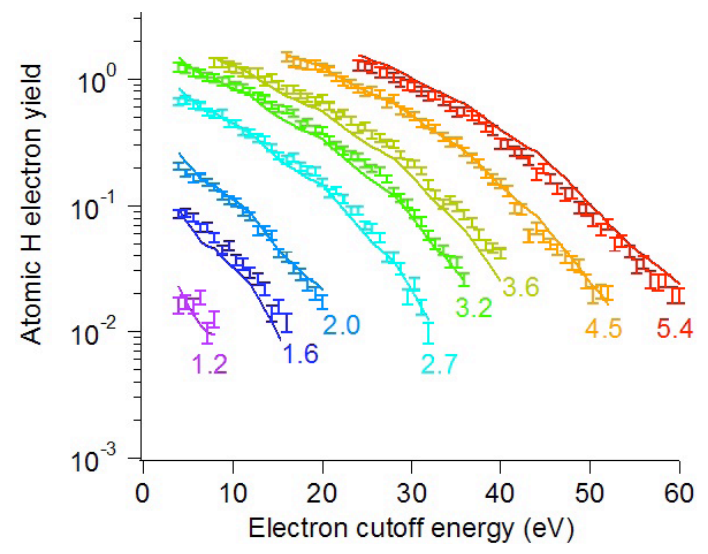

Figure 1. Experimental data (dots) versus theoretical predictions (solid line). Experimental data agrees quantitatively with TDSE calculations at the $10 \%$ level. The laser intensity ranges from 1.2 to 5.4 $\mathrm{x} 10^{14} \mathrm{~W} / \mathrm{cm}^{-2}$.

\section{References}

[1] L.F. DiMauro et al 2009 Nature Phys. 5335

[2] P. Agostini et al 1979 Phys. Rev. Lett. 421127 